\title{
Performance Analysis of LDPC Codes for Time-Selective Complex Fading Channels
}

\author{
Kaiann Fu and Achilleas Anastasopoulos \\ Electrical Engineering and Computer Science Dept. \\ University of Michigan, Ann Arbor, MI 48109-2122
}

\begin{abstract}
Low-density parity-check (LDPC) codes are analyzed using density evolution techniques for a frequency-nonselective, time-selective fading channel where the fading affects both the amplitude and the phase of the transmitted signal. To aid the channel estimation process, pilot symbols are transmitted periodically. The performance of several message-passing estimation/decoding algorithms is investigated, and the optimal energy allocation between the pilot and coded symbols is evaluated. The results show that optimal power allocation can improve the performance by more than $1 \mathrm{~dB}$ for moderate channel dynamics. Finally, necessary and sufficient conditions for the convergence to error-free transmission are derived that take into account the channel coherence time.
\end{abstract}

\section{INTRODUCTION}

Low-density parity-check (LDPC) codes in conjunction with iterative decoding based on message-passing algorithms have been shown to achieve excellent performance over the AWGN channel [1], [2]. Their potential as capacity-achieving codes for more realistic wireless channels has not been established yet. However, experimental evidence as well as some preliminary analytical results [3] have led to the conjecture that LDPC-or in general turbo-like codes-can achieve capacity for a wide range of channels [4]. In particular, the analysis of irregular LDPC codes based on density evolution developed in [1], [2] was extended in [3] for a memoryless frequency-non-selective (i.e., flat) Rayleigh fading channel.

In this paper, we extend the analysis and design of LDPC codes of [3] in two important directions. First, the effects of both the amplitude and the phase of the fading channel are taken into account. This is certainly a more realistic model than the one assuming only amplitude variations of the transmitted signal. Furthermore, when fading affects both the amplitude and the phase of the transmitted symbols, code design becomes significantly more difficult, as evidenced in [5]. ${ }^{1}$ Second, channel dynamics are explicitly taken into account by considering a block-independent fading model. In particular, the complex fading is considered constant for a block of length $N$ (i.e., the channel coherence time) and independent from block to block. Although coding for this channel is generally complicated, in this paper we consider a simple coding scheme, namely pilot-symbol-assisted LDPC codes. A pilot symbol of specified energy is added in the beginning of each block to establish a reference for the phase of the symbols and aid (implicitly) the estimation/decoding process. Multiple pilots could be used in each block, but we only consider the single-pilot per

${ }^{1}$ It is noted that this work refers to the more interesting case of space-time coded complex fading channels. block case. Clearly, the quality of channel estimation improves with increased energy in the pilot, while the quality of the decoded symbols depends on the energy spent on the coded symbols. Thus a trade-off between allocation of energy to the pilots and coded symbols exists. Using density evolution, this tradeoff is studied analytically, and the optimal power allocation is obtained for several receiver structures first suggested and analyzed in [6], [7]. Thus a quantitative answer, that is closely related to a particularly simple family of codes and decoders, is obtained to the question of "how much pilot energy is required for transmission in the noncoherent fading channel?" [8], [9] for the single-antenna transmission scenario. These optimized pilot-symbol-assisted LDPC codes are shown to perform very close to the case when perfect channel state information (CSI) is available at the receiver for slow and moderate channel dynamics, and the optimal allocation depends both on the channel coherence time and the particular receiver used.

The rest of the paper is structured as follows. In Section II, we discuss the system and channel model. The specific message-passing estimation/decoding algorithms are described in Section III. The performance analysis using density evolution is presented in Section IV and the numerical results are discussed in Section V. In Section VI, symmetry and stability conditions are discussed.

\section{System AND Channel Model}

The factor graph [10] of the system considered here is shown in Fig. 1. Each variable node represents a coded symbol, and each code check node represents a parity check. In addition, the variable nodes are connected to channel constraint nodes which

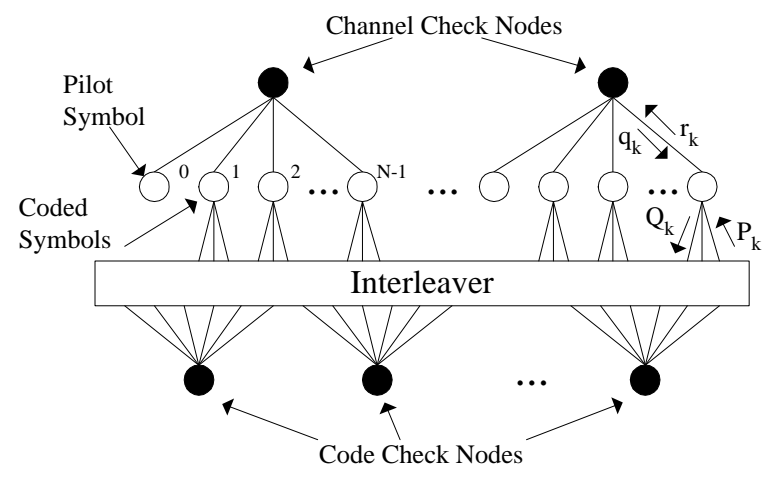

Fig. 1. Factor graph for pilot-symbol-assisted LDPC code in a flat, blockindependent fading channel. 
represent the constraints imposed by the fading channel. The analysis presented in this paper can be applied to general irregular LDPC codes with maximum variable (check) node degree of $d_{v}\left(d_{c}\right)$ and degree polynomials $\lambda(x)$ and $\rho(x)$ given in [2].

To facilitate channel estimation, the first transmitted symbol in each block of length $N$ is a pilot symbol (i.e., $a_{0}=0$ ) of energy $E_{p}$, followed by $N-1$ coded symbols, $a_{k} \in\{0,1\}$ with energy $E_{s}$. The effective energy per information bit is $E_{b}=\left(E_{s}+E_{p} /(N-1)\right) / R$ where $R$ is the rate of the LDPC code. Due to the pilot transmission, the overall rate of the code is $R_{c}=R(N-1) / N$. The received symbols for each block of length $N$ can be expressed as

$$
z_{k}=c \sqrt{E_{k}}(-1)^{a_{k}}+n_{k} \quad k=0, \ldots, N-1,
$$

where $E_{0}=E_{p}$ and $E_{k}=E_{s}$ for $k=1, \ldots, N-1$, the fading coefficient $c$ is modeled as a zero-mean circular complex Gaussian random variable with $E\left[|c|^{2}\right]=1$, and the AWGN noise is modeled by independent zero-mean circular complex Gaussian random variables $n_{k}$ with $E\left[\left|n_{k}\right|^{2}\right]=N_{0}$. Thus, the fading amplitude has a Rayleigh density while the fading phase has a uniform density in $[0,2 \pi)$.

\section{Decoding Algorithms}

Iterative decoding can be accomplished by message-passing algorithms operating on the factor graph of the system. Message exchange between variable and check nodes for LDPC codes is well understood, thus we concentrate on the messages generated at the channel constraint node. In the following, we describe different options for message generation at the channel constraint node.

a) Sum-Product Algorithm: The channel constraint node generates a log-likelihood ratio for the $k$ th variable node based on the information it receives from the $N-1$ other variable nodes and on the $N$ channel output values. Assuming that the incoming message from the $i$ th variable node to the channel constraint node is a log-likelihood ratio of the form $r_{i}=\log \left(p_{i}\left(a_{i}=0\right) / p_{i}\left(a_{i}=1\right)\right),{ }^{2}$ the outgoing message to the $k$ th variable node can be expressed as

$$
q_{k}=\log \frac{\sum_{\mathbf{a}: a_{k}=0} \mathcal{C N}_{N}(\mathbf{z} ; \mathbf{0}, \mathbf{K}(\mathbf{a})) \prod_{i=0, i \neq k}^{N-1} p_{i}\left(a_{i}\right)}{\sum_{\mathbf{a}: a_{k}=1} \mathcal{C N}_{N}(\mathbf{z} ; \mathbf{0}, \mathbf{K}(\mathbf{a})) \prod_{i=0, i \neq k}^{N-1} p_{i}\left(a_{i}\right)},
$$

where $\mathcal{C N}_{N}(\mathbf{z} ; \mathbf{0}, K(\mathbf{a}))$ denotes an $N$-dimensional zeromean complex Gaussian pdf with covariance matrix

$$
\mathbf{K}(\mathbf{a})=E\left[\mathbf{z z}^{H} \mid \mathbf{a}\right]=\boldsymbol{\mu} \boldsymbol{\mu}^{H}+N_{0} \mathbf{I}_{N},
$$

where $\boldsymbol{\mu}=\left(\mu_{0}, \ldots, \mu_{N-1}\right)^{T}, \mu_{i}=\sqrt{E_{i}}(-1)^{a_{i}}$, and $\mathbf{I}_{N}$ is the $N \times N$ identity matrix.

It is a well-known fact that if the factor graph is cycle-free, then at the termination of the sum-product algorithm, the maximum a posteriori (MAP) estimate of each coded symbol is obtained [10]. However, evaluating the channel-to-variable-node

\footnotetext{
${ }^{2}$ It is assumed that the incoming message corresponding to the pilot symbol is $r_{0}=+\infty$.
}

message has exponential complexity, as evidenced by the expression in (2). Motivated by this fact, suboptimal implementations of this operation are suggested in the next paragraphs c) and e). In addition, paragraphs b) and d) deal with special cases that serve as lower bounds on the performance of the sum-product algorithm.

b) Perfect Channel State Information: When perfect CSI is available at the receiver, i.e., when the fading amplitude $c$ is exactly known at the receiver side, the messages $q_{k}$ can be evaluated as in [3]

$$
q_{k}=\log \frac{f\left(z_{k} \mid a_{k}=0, c\right)}{f\left(z_{k} \mid a_{k}=1, c\right)}=\frac{4 \sqrt{E_{s}}}{N_{0}} \operatorname{Re}\left\{z_{k} c^{*}\right\} .
$$

Since the fading coefficient is known, no pilot symbol is necessary. In addition, since $q_{k}$ is independent of all incoming messages $r_{i}$, the message $q_{k}$ does not change with iterations.

c) Pilot Only Detection: In the pilot only (PO) case, only the pilot symbol is used by the channel constraint node to obtain information about the channel. In this case, the message from the channel $q_{k}$ for each coded symbol is defined by

$$
q_{k}=\log \frac{f\left(z_{k}, z_{0} \mid a_{k}=0\right)}{f\left(z_{k}, z_{0} \mid a_{k}=1\right)}=\frac{4 \sqrt{E_{s}} \operatorname{Re}\left\{z_{k} \sqrt{E_{p}} z_{0}^{*}\right\}}{N_{0}\left(N_{0}+E_{p}+E_{s}\right)} .
$$

Similar to the perfect CSI case, the message $q_{k}$ is independent of all messages $r_{i}$, so it is the same at each iteration.

d) Pilot and Data Correct Decision Feedback: Pilot and data correct decision feedback (PDCDF) is not a practical algorithm. However, it can be used to derive a lower bound on the minimum $E_{b} / N_{0}$ required to achieve error-free transmission. In this case, the output of the channel constraint node for the $k$ th symbol is determined by the pilot symbol as well as the other $N-2$ symbols in the block. However, it is assumed that due to the presence of a genie, the correct values of these $N-2$ symbols $\left(a_{1}, \ldots, a_{k-1}, a_{k+1}, \ldots, a_{N-1}\right)$ are available in each iteration. The resulting message is of the form

$$
q_{k}=\frac{4 \sqrt{E_{s}} \operatorname{Re}\left\{z_{k}\left(\sqrt{E_{p}} z_{0}+\sqrt{E_{s}} \sum_{i=1, i \neq k}^{N-1}(-1)^{a_{i}} z_{i}\right)^{*}\right\}}{N_{0}\left(N_{0}+E_{p}+E_{s}(N-1)\right)} .
$$

Once again, it is observed that the messages $q_{k}$ remain the same at each iteration. In addition, as it will be shown in the next section, this is equivalent to the PO case where the effective pilot energy is $E_{p}+(N-2) E_{s}$, since the other $N-2$ symbols in the block also act as pilots for the $k$ th symbol. Finally, following an argument similar to the one used in [6], one can show that the PDCDF receiver performs better than the sum-product algorithm as well.

e) Quantized Decision Feedback: In the PDCDF case, $N-2$ coded symbols in the block act as pilots for calculating the log-likelihood for the $k$ th symbol. This is a hypothetical scenario where the receiver knows exactly the values of the $N-2$ symbols sent. However, when the incoming messages $r_{i}$ are strongly biased towards $\pm \infty$ the corresponding symbols 
can act as pilots as well. Motivated by this observation, we propose an ad-hoc algorithm, quantized decision feedback (QDF), which operates as follows. The incoming messages are first quantized according to the following rule

$$
\hat{r}_{i}=\left\{\begin{aligned}
+1 & \text { if } T<r_{i} \\
0 & \text { if }-T \leq r_{i} \leq T \\
-1 & \text { if } r_{i}<-T
\end{aligned}\right.
$$

where $T$ is a predetermined threshold value. The message $q_{k}$ is now evaluated assuming that all symbols for which $\left|r_{i}\right|>T$ act as pilots, while those for which $\left|r_{i}\right| \leq T$ do not contribute in the estimation process.

$$
q_{k}=\frac{4 \sqrt{E_{s}} \operatorname{Re}\left\{z_{k}\left(\sqrt{E_{p}} z_{0}+\sqrt{E_{s}} \sum_{i=1, i \neq k}^{N-1} \hat{r}_{i} z_{i}\right)^{*}\right\}}{N_{0}\left(N_{0}+E_{p}+E_{s}\left(N^{\prime}+1\right)\right)}
$$

where $N^{\prime}$ is the number of non-zero quantized messages, other than the one corresponding to the $k$ th symbol. It is emphasized that unlike the previous cases, in the QDF scheme, the channel constraint node must recalculate the message $q_{k}$ at each iteration, thus resulting in an iterative joint detection/estimation technique.

\section{Performance Analysis with Density Evolution}

Analysis using density evolution involves evaluating the probability density functions (pdfs) of the messages exchanged between the nodes of the factor graph [1], given the pdfs of the initial messages. To apply density evolution, the all-zero codeword is assumed to have been transmitted. This assumption is not restrictive since the fading channel considered herein satisfies the channel symmetry condition $f\left(z_{k} \mid a_{k}=0\right)=$ $f\left(-z_{k} \mid a_{k}=1\right)$. In addition, for the decoding algorithms described in Section III, the channel constraint node preserves symmetry since a flip in sign of $z_{k}$ results in a flip in sign of $q_{k}$ at each iteration.

Under the standard assumptions of large girth (compared to the iteration number), the neighborhood of a graph is essentially a tree. In this case, all messages passed in the factor graph are independent and all calculated pdfs are exact. In the practical situation where cycles are present in the graph, it was shown in [1] that the average behavior of the code converges to the cycle-free case as the length of the code increases.

It is observed that for all algorithms (except the QDF algorithm) described in Section III, the messages from the channel constraint nodes do not change with iterations. Thus, in order to perform density evolution in these cases, it suffices to evaluate the initial message densities and follow the standard pdf transformations described in [1] to trace the pdfs of the messages exchanged in the code portion of the factor graph. Furthermore, it can be observed that all $q_{k}$ messages described previously are of the form

$$
q=C \operatorname{Re}\left\{x y^{*}\right\}
$$

where $C$ is a constant, $x$ and $y$ are zero-mean complex Gaussian variables, and the subscript $k$ is dropped for notational simplicity. The pdf of $q$ can be found using the results in Appendix $B$ of [11] as

$$
f(q)=\frac{v_{1} v_{2}}{v_{1}+v_{2}}\left[e^{v_{2} q} u(-q)+e^{-v_{1} q} u(q)\right]
$$

where $u(q)$ is the unit step function, and $v_{1}$ and $v_{2}$ are given by

$$
\begin{aligned}
& v_{1}=1 /\left(C\left(\sqrt{\mu_{x x} \mu_{y y}}+\mu_{x y}\right)\right) \\
& v_{2}=1 /\left(C\left(\sqrt{\mu_{x x} \mu_{y y}}-\mu_{x y}\right)\right),
\end{aligned}
$$

where $\mu_{x x}=\frac{1}{2} E\left[x x^{*}\right], \mu_{y y}=\frac{1}{2} E\left[y y^{*}\right]$, and $\mu_{x y}=\frac{1}{2} E\left[x y^{*}\right]$.

a) The Perfect CSI, PO, and PDCDF Receivers: In the perfect CSI case, $v_{1}$ and $v_{2}$ are given by

$$
\begin{aligned}
& v_{1}=N_{0} /\left(2 \sqrt{E_{s}}\left(\sqrt{E_{s}+N_{0}}+\sqrt{E_{s}}\right)\right) \\
& v_{2}=N_{0} /\left(2 \sqrt{E_{s}}\left(\sqrt{E_{s}+N_{0}}-\sqrt{E_{s}}\right)\right) .
\end{aligned}
$$

Substituting these results in (10) we get a closed form expression for the initial density, which is the same result as in [3].

Similarly, for the PO case, the corresponding constants are evaluated as

$$
\begin{aligned}
& v_{1}=\frac{N_{0}\left(E_{p}+E_{s}+N_{0}\right)}{2 \sqrt{E_{p} E_{s}}\left(\sqrt{\left(E_{p}+N_{0}\right)\left(E_{s}+N_{0}\right)}+\sqrt{E_{p} E_{s}}\right)} \\
& v_{2}=\frac{N_{0}\left(E_{p}+E_{s}+N_{0}\right)}{2 \sqrt{E_{p} E_{s}}\left(\sqrt{\left(E_{p}+N_{0}\right)\left(E_{s}+N_{0}\right)}-\sqrt{E_{p} E_{s}}\right)}
\end{aligned}
$$

The message pdfs for the hypothetical PDCDF receiver can be evaluated using (9) with $x=z_{k}$ and $y=\sqrt{E_{p}} z_{0}+$ $\sqrt{E_{s}} \sum_{i=1, i \neq k}^{N-1}(-1)^{a_{k}} z_{i}$, resulting in equations similar to (13) with $E_{p}$ substituted by the effective pilot energy $E_{p, e f f}=$ $E_{p}+(N-2) E_{s}$.

b) The QDF Receiver: For the QDF receiver, the message pdfs at the $l$ th iteration from the channel constraint node are dependent on the pdfs $f^{(l-1)}\left(r_{i}\right)$ of the incoming messages $r_{i}$ at the previous iteration. The message pdf from the channel constraint node at the $l$ th iteration can be evaluated using total probability as

$$
f^{(l)}(q)=\sum_{g=0}^{N-2} \sum_{e=0}^{N-2-g} f(q \mid g, e) p^{(l-1)}(g, e)
$$

where $g$ is the number of good messages with $\hat{r}_{i}=+1$ and $e$ is the number of erroneous messages with $\hat{r}_{i}=-1$. For the initial message density, $\hat{r}_{i}=0$ for all $i$. Furthermore, the joint mass function $p^{(l-1)}(g, e)$ of good and erroneous quantized signals at the $l-1$ st iteration is given by a multinomial distribution

$$
p^{(l-1)}(g, e)=\left(\begin{array}{c}
N-2 \\
g, e
\end{array}\right) p_{g}^{g} p_{e}^{e}\left(1-p_{g}-p_{e}\right)^{N-2-g-e}
$$

where $p_{g}=P\left(\hat{r}_{i}=+1\right)=\int_{T}^{\infty} f^{(l-1)}\left(r_{i}\right) d r_{i}$ and $p_{e}=$ $P\left(\hat{r}_{i}=-1\right)=\int_{-\infty}^{-T} f^{(l-1)}\left(r_{i}\right) d r_{i}$. The conditional pdfs 
$f(q \mid g, e)$ can be evaluated using (9) and (10) with $x=z_{k}$ and $y=\sum_{i=0, i \neq k}^{N-1} \sqrt{E_{i}} \hat{r}_{i} z_{i}$. The constants $v_{1}$ and $v_{2}$ are determined using (11) with the following relationships

$$
\begin{aligned}
& E\left[x x^{*}\right]=E_{s}+N_{0} \\
& E\left[y y^{*}\right]=\left(E_{p}+E_{s}(g-e)\right)^{2}+N_{0}\left(E_{p}+E_{s}(g+e)\right)
\end{aligned}
$$

$$
E\left[x y^{*}\right]=\sqrt{E_{s}}\left(E_{p}+E_{s}(g-e)\right)
$$

\section{NumericAl RESUlts}

In this section, density evolution results are generated using discretized density evolution [12]. In particular, each message is quantized using an $R_{Q}$-bit uniform quantizer with $2^{R_{Q}}-1$ quantization levels. The range of the quantizer is very roughly optimized to obtain low $E_{b} / N_{0}$ thresholds for reasonable values of $R_{Q}$. Since messages are quantized, only probability mass functions need to be evaluated, thus the resulting $E_{b} / N_{0}$ thresholds can be considered as upper bounds for belief propagation with continuous densities. However, discretized density evolution is exact for a practical receiver that quantizes the messages before iterative processing.

The following results are all based on a $\left(d_{v}, d_{c}\right)=(3,6)$ regular LDPC code and discretized density evolution using an 8 -bit quantizer in the range $(-35,35)$. A binary search over $E_{b} / N_{0}$ with a resolution of $0.001 \mathrm{~dB}$ is conducted to find the smallest $E_{b} / N_{0}$ such that the bit error rate is less than $10^{-8}$ in at most 1000 iterations. In addition, the distribution of power to the pilot and coded symbols was numerically optimized (for the PO and QDF receivers) to minimize the $E_{b} / N_{0}$ threshold.

Fig. 2 displays a summary of the density evolution results for the different decoding schemes. For the perfect CSI case, density evolution resulted in an $E_{b} / N_{0}$ threshold of $3.06 \mathrm{~dB}$, which agrees with the results in [3]. We note that for the perfect CSI case, the overall code rate $R_{c}=R=0.5$. However, for the other cases where a pilot symbol is transmitted, $R_{c}=R(N-1) / N=0.5(N-1) / N$. Thus, the comparison between these cases with the CSI case is not exactly fair, especially for small values of $N$. This inconsistency can be corrected by using different irregular LDPC codes with appropriate degree polynomials such that the overall rates of the compared systems are equal.

In the same figure, the PDCDF curve is shown as a lower bound on the $E_{b} / N_{0}$ threshold. It is observed that for large $N$, the performance of both the PO and PDCDF cases approaches the perfect CSI performance, which can also be proved using (12), and (13).

The performance gain when the energy distribution between $E_{p}$ and $E_{s}$ is optimized can also be observed in Fig. 2. For $N=20$, the threshold for the PO receiver is about $1.7 \mathrm{~dB}$ lower with the energy distribution optimized compared to the case when $E_{p}=E_{s}$. A $0.8 \mathrm{~dB}$ improvement is seen in the equivalent scenario for the QDF receiver. The optimal values of $E_{p} / E_{s}$ for $N=10$ and $N=20$ are $5.4 \mathrm{~dB}$ and $7.2 \mathrm{~dB}$,

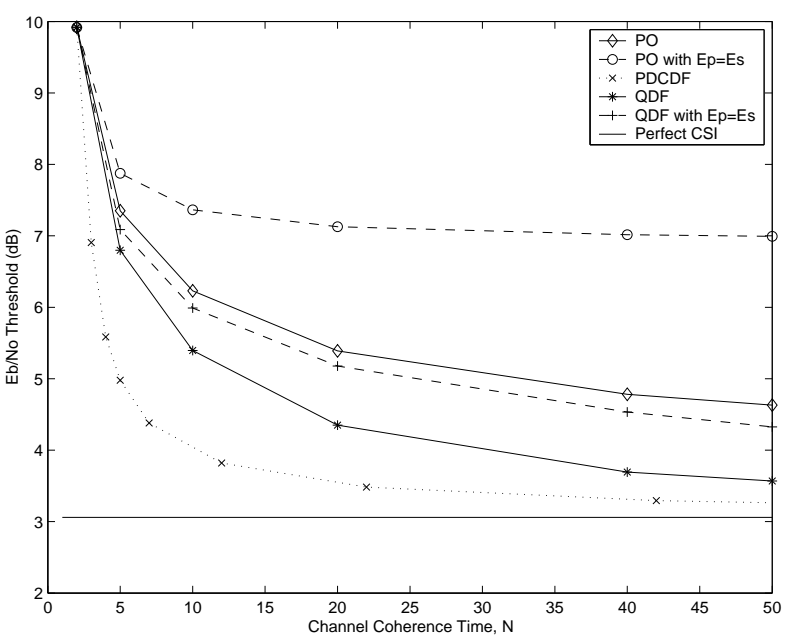

Fig. 2. Discretized density evolution results for $(3,6)$ regular LDPC code over a complex Gaussian flat fading channel.

respectively, for the $\mathrm{PO}$ receiver and $3.5 \mathrm{~dB}$ and $3.3 \mathrm{~dB}$ for the QDF receiver.

The performance of the QDF receiver is significantly better than the PO receiver. For $N=20$, the $E_{b} / N_{0}$ threshold for the optimized QDF case is about $4.4 \mathrm{~dB}$ which is about $1.0 \mathrm{~dB}$ lower than the optimized $\mathrm{PO}$ threshold and $0.8 \mathrm{~dB}$ higher than the PDCDF lower bound. For $N=40$, the QDF threshold is $3.7 \mathrm{~dB}$ which is about $1.1 \mathrm{~dB}$ lower than the PO case and $0.4 \mathrm{~dB}$ away from the PDCDF lower bound. The gap between the QDF and PDCDF performance decreases as $N$ increases.

It should be emphasized that for small values of the channel coherence time $N$, i.e., $N=5$ to 10 , the performance difference between the optimized QDF and the lower bound is quite significant $(\approx 2 \mathrm{~dB})$. At this point, it is not clear whether this is a limitation of the pilot-symbol-assisted scheme, or a limitation of the suboptimal QDF receiver, or both, nor is it clear whether the PDCDF bound is tight in this region.

\section{Symmetry and Stability Conditions}

In this section, we will discuss necessary and sufficient conditions for convergence (to zero probability of error) of the iterative schemes described earlier. The basic result in this area was derived in [2] for LDPC codes operating on binaryinput/scalar-output memoryless channels. In particular, it was shown that if the channel input/output description is symmetric, i.e., if the pdf of the scalar output $y$, conditioned on the binary input $a$ satisfies

$$
f(y \mid a=0)=f(-y \mid a=1),
$$

then the pdf $f(q)$ of the initial message $q=\log \frac{f(y \mid a=0)}{f(y \mid a=1)}$ satisfies an exponential symmetry of the form

$$
f(-q)=e^{-q} f(q) \quad q>0 .
$$

Furthermore, it was shown that this exponential symmetry is invariable to message transformations related to the sum-product 
algorithm. Based on this result, necessary and sufficient conditions that involve the degree polynomials and the channel parameters were derived that guarantee the convergence of the sum-product algorithm to zero probability of error. Specifically, the general stability condition stated in [2] is as follows. For an exponential symmetric initial message density $f(q)$, define

$$
s=-\log \int_{-\infty}^{\infty} f(q) e^{-q / 2} d q
$$

If $\lambda^{\prime}(0) \rho^{\prime}(1)>e^{s}$, then the probability of error will be bounded away from zero at all iterations. If $\lambda^{\prime}(0) \rho^{\prime}(1)<e^{s}$, then there exists an $\epsilon>0$ such that $\int_{-\infty}^{0} f(q) d q<\epsilon$ implies that the probability of error converges to zero as the number of iterations increases to infinity.

There are two main obstacles in applying the above results to the algorithms presented in Section III: (i) the channels under consideration are not memoryless, and (ii) the channel output is not a scalar, but a complex vector. Regarding the first obstacle, the requirement of a memoryless channel is not actually necessary if the decoding algorithm and the channel satisfy the following conditions: the messages from the channel constraint node do not change with iterations and all messages arriving at a given variable or code check node are independent at each iteration. It can be shown for the PO algorithm that the channel is equivalent to a binary-input vector-output channel where the input $a_{k}$ results in a channel output $\left[z_{k}, z_{0}\right]$. This case satisfies the above conditions as long as the code is long enough and the interleaver is chosen suitably. In the following we present a method that overcomes the second obstacle by transforming the vector channel to an equivalent scalar channel with input/output symmetry as in (17). In particular, consider a new channel which takes the output of the original channel, $\left[z_{k}, z_{0}\right]$, and creates a new output

$$
y_{k}=\log \frac{f\left(z_{k}, z_{0} \mid a_{k}=0\right)}{f\left(z_{k}, z_{0} \mid a_{k}=1\right)} .
$$

It should be clear that $y_{k}$ is a sufficient statistic for $a_{k}$ since no information is lost in the new channel. Thus, the concatenation of the original and new channels forms an equivalent binaryinput scalar-output channel (from $a_{k}$ to $y_{k}$ ). Furthermore, the density $f\left(y_{k} \mid a_{k}=0\right)$ is in the form of (10) where $v_{1}$ and $v_{2}$ are expressed in (13), and the density $f\left(y_{k} \mid a_{k}=1\right)$ is the same as $f\left(y_{k} \mid a_{k}=0\right)$ except that $v_{1}$ and $v_{2}$ are switched. Thus, the equivalent channel satisfies the input/output symmetry of (17). As a result, the corresponding initial message $q_{k}^{\prime}$ used by the sum-product algorithm,

$$
\begin{aligned}
q_{k}^{\prime} & =\log \frac{\frac{v_{1} v_{2}}{v_{1}+v_{2}}\left[e^{v_{2} y_{k}} u\left(-y_{k}\right)+e^{-v_{1} y_{k}} u\left(y_{k}\right)\right]}{\frac{v_{1} v_{2}}{v_{1}+v_{2}}\left[e^{v_{1} y_{k}} u\left(-y_{k}\right)+e^{-v_{2} y_{k}} u\left(y_{k}\right)\right]} \\
& =\left(v_{2}-v_{1}\right) y_{k}=\left(v_{2}-v_{1}\right) \frac{4 \sqrt{E_{s}} \operatorname{Re}\left\{z_{k} \sqrt{E_{p}} z_{0}^{*}\right\}}{N_{0}\left(N_{0}+E_{p}+E_{s}\right)},
\end{aligned}
$$

will satisfy the exponential symmetry of (18). Thus the stability conditions of [2], stated in (19), hold for the PO case, with the quantity $e^{s}$ evaluated as

$$
e^{s}=1+\frac{E_{p} E_{s}}{N_{0}\left(E_{p}+E_{s}+N_{0}\right)},
$$

which implicitly depends on the channel coherence time $N$. Furthermore, since the PDCDF case is equivalent to the PO case with an effective pilot energy of $E_{p, e f f}=E_{p}+(N-2) E_{s}$, these results also apply to the PDCDF case. Finally, for the perfect CSI case, the corresponding result can be found to be $e^{s}=1+E_{s} / N_{0}$, which agrees with the result of [3].

\section{CONCLUSIONS}

In this paper, we have extended the analysis of LDPC codes to frequency-non-selective, time-selective fading channels where the fading affects both the amplitude and phase of the transmitted symbols and the channel dynamics are taken explicitly into account. Two practical receiver algorithms were proposed (i.e., the PO and QDF receivers) and analyzed using density evolution. Using this analytical tool, the allocation of power to the pilots and the coded symbols was optimized. Results showed that the performance of the optimized codes is 1 to $2 \mathrm{~dB}$ better than the non-optimized ones. Finally, by transforming the PO receiver to an equivalent binary-input/scalar-output symmetric channel, stability conditions similar to the ones derived in [2] were obtained.

\section{REFERENCES}

[1] T. J. Richardson and R. L. Urbanke, "The capacity of low-density paritycheck codes under message-passing decoding," IEEE Trans. Information Theory, vol. 47, pp. 599-618, Feb. 2001.

[2] T. J. Richardson, M. A. Shokrollahi, and R. L. Urbanke, "Design of capacity-approaching irregular low-density parity-check codes," IEEE Trans. Information Theory, vol. 47, pp. 619-637, Feb. 2001.

[3] J. Hou, P. H. Siegel, and L. B. Milstein, "Performance analysis and code optimization of low-density parity-check codes on Raleigh fading channels," IEEE J. Select. Areas Commun., vol. 19, pp. 924-934, May 2001.

[4] R. J. McEliece, "The effectiveness of turbolike codes on nonstandard channel models," in Proc. International Symposium on Information Theory, (Washington, D.C.), June 2001. Plenary talk.

[5] T. L. Marzetta and B. Hochwald, "Capacity of a mobile multiple-antenna communication link in Rayleigh flat fading," IEEE Trans. Information Theory, vol. 45, pp. 139-157, Jan. 1999.

[6] R. Nuriyev and A. Anastasopoulos, "Analysis and design of pilotsymbol-assisted codes, for the non-coherent AWGN channel, using density evolution," in Proc. International Conf. Communications, (New York, NY), pp. 1511-1515, Apr. 2002.

[7] R. Nuriyev and A. Anastasopoulos, "Analysis of joint iterative decoding and phase estimation for the non-coherent AWGN channel, using density evolution," in Proc. International Symposium on Information Theory, (Lausanne, Switzerland), p. 168, June 2002.

[8] B. Hassibi and B. Hochwald, "How much training is needed for multipleantenna wireless links," IEEE Trans. Information Theory. (submitted for publication).

[9] L. Zheng and D. N. C. Tse, "Communication on the Grassmann manifold: a geometric approach to the noncoherent multiple-antenna channel," IEEE Trans. Information Theory, vol. 48, pp. 359-383, Feb. 2002.

[10] B. J. Frey, Graphical models for machine learning and digital communications. Cambridge, MA: MIT Press, 1998.

[11] J. G. Proakis, Digital Communications. New York: McGraw-Hill, 3rd ed., 1995.

[12] S.-Y. Chung, G. D. Forney, Jr., T. J. Richardson, and R. L. Urbanke, "On the design of of low-density parity-check codes within $0.0045 \mathrm{~dB}$ of the Shannon limit," IEEE Commun. Lett., vol. 5, pp. 58-60, Feb. 2001. 